\title{
External Upper Lip
}

National Cancer Institute

\section{Source}

National Cancer Institute. External Upper Lip. NCI Thesaurus. Code C12221.

The external surface of the upper lip. 